\title{
Modelo de dinámica de sistemas para la modelación de cadenas de Markov de tiempo Continuo - No homogéneas
}

\author{
System Dynamics Model for the modeling of Nonhomogeneous Continous-Time \\ Markov Chains
}

\author{
M. Narváez-Velasco iD; J. C. Osorio-Gómez iD
}

\begin{abstract}
This paper treats with two modeling techniques: Nonhomogeneous Continuous-Time Markov Chains (NHCTMC) and Systems Dynamics (SD). In general, each of these focuses on different types of problems. While the NH-CTMCare used in the modeling of stochastic processes, the SD is focused on modeling a system from functional relationships between its components. However, it is demonstrated that the concepts and tools of the SD can be effectively used in modeling of NH-CTMC by means of the building and simulating an Equivalent Systems Dynamics Model (ESDM) representing the state probabilities over time. The ESDMis based on mathematical equivalence between the two models, which is shown through an example and relevant considerations in its application are summarized. This proposal could be used to study reliability in complex systems using more simple calculations.
\end{abstract}

Index Terms - Equivalent Systems Dynamics Model; Flow equation; Intensity function; Level equation; Nonhomogeneous Continuous-Time Markov Chains; State probability; Systems Dynamics.

Resumen-El presente trabajo trata con dos técnicas de modelación: las Cadenas de Markov de Tiempo Continuo - No Homogéneas (CMTC-NH) y la Dinámica de Sistemas (DS). En general, cada una se concentra en diferentes tipos de problemas. Mientras las CMTC-NH se emplean en la modelación de procesos estocásticos, la DS se concentra en la modelación de un sistema a partir de las relaciones funcionales entre sus componentes. Sin embargo, se demuestra que los conceptos y las herramientas de la DS pueden ser eficazmente empleados en la modelación de CMTC-NH, mediante la construcción y la simulación de un Modelo de Dinámica de Sistemas Equivalente (MDSE), que representa las probabilidades de estado a través del tiempo. Dicho MDSE se basa en la equivalencia matemática encontrada entre ambos tipos de modelos, la cual se demuestra a través de un ejemplo y se presentan consideraciones pertinentes en su aplicación. La utilidad de este trabajo está en la posibilidad de

Este manuscrito fue enviado el 31 de agosto de 2019 y aceptado el 8 de marzo de 2020

M. Narváez-Velasco, Ingeniera Industrial egresada de la Universidad del Valle, Magister en Finanzas de la Universidad Icesi, Santiago de Cali, Colombia.

J. C. Osorio-Gómez, Ingeniero Industrial, Doctor en Ingeniería Industrial de la Universidad del Valle, Santiago de Cali, Colombia, y profesor titular de la Escuela de Ingeniería Industrial utilizar el MDSE para resolver sistemas complejos y evaluar por ejemplo la confiabilidad de los mismos, de manera que se simplifiquen los cálculos y por tanto se faciliten los procesos matemáticos para dichos estudios.

Palabras claves - Modelo de dinámica de sistemas equivalente; Cadena de Markov de Tiempo Continuo - No Homogénea; Dinámica de Sistemas, Ecuación de flujo; Ecuación de nivel; Función de intensidad; Probabilidad de estado.

Resumo - Este artigo trata de duas técnicas de modelagem: Cadeias de Markov contínuas não homogêneas (CMTC-NH) e Dinâmica de sistemas (DS). Em geral, cada um se concentra em diferentes tipos de problemas. Enquanto o CMTC-NH é usado na modelagem de processos estocásticos, o DS se concentra na modelagem de um sistema com base nas relações funcionais entre seus componentes. No entanto, é demonstrado que os conceitos e ferramentas do DS podem ser efetivamente utilizados na modelagem do CMTC-NH, através da construção e simulação de um Modelo Dinâmico de Sistema Equivalente (MDSE), que representa as probabilidades de estado através do tempo. Este MDSE é baseado na equivalência matemática encontrada entre os dois tipos de modelos, demonstrada através de um exemplo e considerações relevantes são apresentadas em sua aplicação. Esta proposta poderia ser usada para estudar a confiabilidade em sistemas complexos usando cálculos mais simples.

Palavras-chave - Cadeia de Markov de tempo contínuo - Não homogênea, Dinâmica de sistemas, Dinâmica de sistemas equivalente, Equação de fluxo, Equação de nível, Função de intensidade, Modelo, Probabilidade de status.

\section{INTRODUCCIÓN}

$\mathrm{E}^{\mathrm{s}}$ $\mathrm{N}$ términos generales, la Dinámica de Sistemas (DS) es una metodología para analizar un sistema que incluye relaciones lógicas y bucles de retroalimentación [1]. Los dos conceptos fundamentales en un Modelo de Dinámica de Sistemas (MDS), son los de variables de nivel y de flujo, cuyo significado es propio de la naturaleza y de los elementos del sistema que se representa [2].

Por su parte, un Modelo Markoviano (MM) representa una secuencia aleatoria de eventos, en la cual el comportamiento probabilístico futuro del proceso no es influenciado por su 
pasado. Los dos conceptos principales corresponden a los de estado y transición, entendido el primero como una condición del sistema, y el segundo como el paso de una condición a otra [3].

Normalmente los MDS y los MM, se consideran métodos de modelación de origen y funciones diferentes. Mientras la DS se concentra en sistemas con relaciones determinísticas, los MM tratan con problemas donde interesa el comportamiento estocástico futuro [4].

De ahí que se encuentren pocos trabajos preocupados por establecer una relación entre ambas metodologías. La primera referencia al respecto es dada por Sahin [5] quién se refirió al supuesto markoviano implícito en muchos modelos de DS al permitir que las ecuaciones de flujo fuesen funciones de las variables de nivel. Ante esto, en su siguiente trabajo Sahin [4] se preocupó por presentar una equivalencia matemática entre las Cadenas de Markov de Tiempo Discreto Homogéneas y los MDS. A pesar de su aporte, también señala la necesidad de extender dicha equivalencia a los modelos no estacionarios o semi-markovianos y plantear un desarrollo formal al respecto.

Más tarde Wood [6] procuró mostrar de qué manera los eventos discretos pueden ser modelados a través de la DS. Sin embargo, a pesar de sus ejemplos, no presenta la formulación matemática detrás de dichos modelos. Un alcance similar lo obtuvieron Mosekilde y Rasmussen [7] quienes se preocuparon por mostrar mediante ejemplos, cómo el software Dynamo podía ser empleado en simular diferentes tipos de procesos estocásticos, entre ellos los MM Homogéneos.

A pesar del avance anterior, ningún otro trabajo se encuentra sobre el tema, hasta el realizado por [5]. Su trabajo es el más completo al demostrar la equivalencia estructural adyacente a los MM Homogéneos y a los MDS de primer orden. Justificando dicha equivalencia, analizan las similitudes y las diferencias entre las dos metodologías, desarrollan ejemplos y comparan los resultados de las simulaciones en el software Dynamo, con los estimados por las expresiones teóricas para cada uno de ellos.

Casi dos décadas después de Mohapatra y Roy [8], su trabajo es citado por Rao y Naikan [9], quienes presentaron un enfoque que llaman Dinámica de Sistemas Markoviano, en el que combinan los MM con la DS para realizar análisis de fiabilidad de un sistema no reparable con un único componente. Sin embargo, por las características del sistema, se trata de un problema relativamente sencillo además de incluir el supuesto de homogeneidad.

En los trabajos antes señalados, aunque se evidencia preocupación por demostrar la aplicabilidad de relaciones entre los MM y los MDS, los fundamentos matemáticos detrás de ellas son débiles y se concentran básicamente en modelos homogéneos. El presente trabajo presenta la teoría asociada a un caso general de MM, las Cadenas de Markov de Tiempo Continuo - No Homogéneas (CMTC-NH), y la relacionada a la DS, con el fin de formular una equivalencia entre ambas metodologías, y construir unModelo de Dinámica de Sistemas
Equivalente (MDSE). Dicho modelo permite analizar el MMa través de la simbología y las herramientas de la DS.

La propuesta se ejemplifica en la construcción de una CMTC-NH con dos estados y funciones de intensidad lineales con el tiempo. Los resultados obtenidos a través del MDSE de dicho sistema, se comparan con la solución analítica correspondiente. Por último, se presentan algunas consideraciones sobre los riesgos y las ventajas del modelo presentado, en términos prácticos y teóricos de su aplicación.

\section{CAdena de MARKov DE TIEMPO CONTINUO (CMTC)}

De acuerdo con Ross [10] un proceso estocástico de tiempo continuo $\{\mathrm{X}(\mathrm{t}), \mathrm{t} \geq 0\}$ que toma valores en un conjunto finito de estados $\mathrm{E}$, es una CMTC si para todo $\mathrm{t} \geq 0$ y estados $\mathrm{j}, \mathrm{i}$, $\mathrm{x}(\mathrm{u}), 0 \leq \mathrm{u}<\mathrm{t}, \quad$ su función deprobabilidad condicional satisface:

$\operatorname{Pr}\{\mathrm{X}(\mathrm{t}+\Delta \mathrm{t})=\mathrm{i} \mid \mathrm{X}(\mathrm{t})=\mathrm{j}, \mathrm{X}(\mathrm{u})=\mathrm{x}(\mathrm{u}), 0 \leq \mathrm{u}<\mathrm{t}\}=$ $\mathrm{p}_{\mathrm{ji}}(\mathrm{t}, \mathrm{t}+\Delta \mathrm{t}) \quad(1)$

Con $\mathrm{p}_{\mathrm{ji}}(\mathrm{t}, \mathrm{t}+\Delta \mathrm{t})=\operatorname{Pr}\{\mathrm{X}(\mathrm{t}+\Delta \mathrm{t})=\mathrm{i} \mid \mathrm{X}(\mathrm{t})=\mathrm{j}\}, \quad$ llamada probabilidad de transición e indica la probabilidad de que el proceso, estando en el estado $\mathrm{i}$ en un instante de tiempo $t$, visite el estado $\mathrm{j}(\mathrm{i} \rightarrow \mathrm{j}$ ), $\Delta$ t unidades de tiempo más tarde.

De acuerdo con Tijms [3] la independencia $\mathrm{p}_{\mathrm{ji}}(\mathrm{t}, \mathrm{t}+\Delta \mathrm{t})$ con $0 \leq \mathrm{u}<\mathrm{t}$, implica la conocida Propiedad Markoviana, según la cual la distribución condicional de un estado futuro, dado el estado presente y los estados pasados de un proceso estocástico $\{\mathrm{X}(\mathrm{t}), \mathrm{t} \geq 0\}$ depende solamente del estado presente $\mathrm{y}$ es independiente del pasado. De ahí la definición dada por (1).

Se dice además que, si $\operatorname{Pr}\{\mathrm{X}(\mathrm{t}+\Delta \mathrm{t})=\mathrm{i} \mid \mathrm{X}(\mathrm{t})=\mathrm{j}\}$ es independiente de $\mathrm{t}$, entonces la CMTC tiene probabilidades de transición estacionarias, y se denomina Cadena de Markov de Tiempo Continuo - Homogénea (CMTC-H). De lo contrario, es decir, cuando $\operatorname{Pr}\{\mathrm{X}(\mathrm{t}+\Delta \mathrm{t})=\mathrm{i} \mid \mathrm{X}(\mathrm{t})=\mathrm{j}\}$ depende del par $(t, t+\Delta t)$ y no solo de la diferencia entre ellos $\Delta t$, se trata de una Cadena de Markov de Tiempo Continuo - No Homogénea (CTMC-NH) [11] .

\section{A. Cadena de Markov de Tiempo Continuo - No Homogenea}

Un proceso estocástico $\{\mathrm{X}(\mathrm{t}), \mathrm{t} \geq 0\}$ evaluado en $\mathrm{E}$ es una CMTC-NH si existe una función de transición matricial no homogénea $\quad\left\{\mathbf{P}(\mathrm{t}, \mathrm{t}+\Delta \mathrm{t})=\left(\mathrm{p}_{\mathrm{ji}}(\mathrm{t}, \mathrm{t}+\Delta \mathrm{t})\right), 0 \leq \mathrm{t} \leq \mathrm{t}+\Delta \mathrm{t}\right\}$ tal que, para todo $\mathrm{j}, \mathrm{i}, \mathrm{x}(\mathrm{u}) 0 \leq \mathrm{u}<\mathrm{t}$ perteneciente a $\mathrm{E}$, se satisface (1).

Cada familia de matrices estocásticas $\mathbf{P}(\mathrm{t}, \mathrm{t}+\Delta \mathrm{t})$ con $0 \leq$ $\mathrm{t} \leq \mathrm{t}+\Delta \mathrm{t}$, se dice que es una función de transición matricial no homogénea si cumple, entre otras condiciones, que $\mathbf{P}(t, t)=\mathbf{I}$ para todo $\mathrm{t} \geq 0$, siendo I una matriz de identidad. Sin embargo, $\{\mathrm{X}(\mathrm{t}), \mathrm{t} \geq 0\}$ puede ser totalmente descrito por una función de intensidad matricial $\{\mathbf{A}(\mathrm{t}), \mathrm{t} \geq 0\}$ definida como se muestra en (2):

$$
\mathbf{A}(\mathrm{t})=\lim _{t+\Delta t \rightarrow t} \frac{P(t, t+\Delta t)-I}{(t+\Delta t)-t}
$$


Si $E=\{1,2, \ldots, k\}, \mathbf{A}(\mathrm{t})=\left(\mathrm{a}_{\mathrm{ji}}(\mathrm{t})\right)$ una matriz $\mathrm{kxk}$, cuyas componentes cumplen que para todo $\mathrm{i}, \mathrm{j} \in \mathrm{E}$ y $\mathrm{t} \geq 0$ :

- $a_{j i}(t) \geq 0$ para $j \neq i$

- $\mathrm{a}_{\mathrm{ii}}(\mathrm{t})=-\sum_{\substack{\mathrm{j} \neq \mathrm{i} \\ \mathrm{j}}}^{\mathrm{k}} \mathrm{a}_{\mathrm{ij}}(\mathrm{t})$ (3)

$\mathrm{a}_{\mathrm{ji}}(\mathrm{t})$ representa la probabilidad instantánea (con $\Delta \mathrm{t} \rightarrow$ 0 ) de visitar el estado i en $t+\Delta t$, dado que el proceso se encontraba en el estado $\mathrm{j}$ en el instante de tiempo $t$.

De acuerdo con (2), la característica principal de una CMTC-NH, es la posibilidad de $\mathrm{a}_{\mathrm{ji}}(\mathrm{t})$ ser cualquier función arbitraria det, y no sólo constante como ocurre en el caso homogéneo. Esta característica es importante en muchos sistemas, en los cuales el tiempo implica, por ejemplo, un proceso de degradación con el tiempo transcurrido, o cierto patrón de comportamiento en el número de llegadas o salidas por unidad de tiempo. En este tipo de casos los MM Homogéneos no son apropiados, y por tanto, las CMTC-NH resultan más adecuadas.

\section{B. Ecuaciones de estado}

De acuerdo a Rausand y Hoyland [12](2004) la distribución de probabilidad $\overrightarrow{\mathrm{P}}(\mathrm{t})=\left[\mathrm{P}_{0}(\mathrm{t}), \ldots, \mathrm{P}_{\mathrm{i}}(\mathrm{t}), \ldots, \mathrm{P}_{\mathrm{r}}(\mathrm{t})\right] \mathrm{de} \quad$ una CMTC-NH, es el conjunto de probabilidades de estado $P_{i}(t)$ con $\mathrm{i}=0,2, \ldots, \mathrm{k}-1$, que representan la probabilidad de que la CMTC-NH se encuentre en el estado i en el instante $t$, y que satisfacen

$$
\sum_{\mathrm{i}=1}^{\mathrm{k}} \mathrm{P}_{\mathrm{i}}(\mathrm{t})=1(4)
$$

Es necesario establecer la condición inicial del proceso estocástico. Si se define que $\mathrm{X}(0)=\mathrm{i}$, de manera que $\mathrm{P}_{\mathrm{i}}(0)=$ $\operatorname{Pr}(X(0)=i)=1$ y $P_{j}(0)=\operatorname{Pr}(X(0)=j)=0$ para $j \neq i, P(t)$ se puede encontrar al resolver el siguiente sistema de ecuaciones diferenciales:

$$
\frac{d P_{i}(t)}{d t}=\sum_{j=1}^{k} a_{j i}(t) P_{j}(t) \quad \forall i \in E
$$

La suma en fila de los componentes deA(t) es igual a 0 , de manera que $\mathbf{A}(\mathrm{t})$ es una matriz singular, y como consecuencia, el sistema correspondiente a (5) es linealmente dependiente. Por lo tanto, es necesario construir un sistema compuesto por las (5) junto con la definida por (4), cuya independencia garantiza una solución única [13].

\section{Probabilidades en el límite}

La probabilidad de que la CMTC-NH estará en el estado i en el instante de tiempo $t$ converge hacia un valor límite $\mathrm{P}_{\mathrm{i}}$ el cual es independiente del estado inicial y se describe como [10],

$$
P_{i}=\lim _{\Delta t \rightarrow \infty} p_{j i}(t, t+\Delta t)(6)
$$

$\mathrm{P}_{\mathrm{i}}$, es denominada probabilidad de estado estacionario, $\mathrm{y}$ puede ser interpretada como la proporción del tiempo que el proceso estará en el estado i en el largo plazo. Cuando esta condición de estacionariedad no se ha alcanzado y las probabilidades de estado aun dependen del instantet, se dice que el proceso se encuentra en estado transitorio [3].

\section{Determinación del $\widehat{t}^{*}$}

Para efectos del presente trabajo se denomina $t^{*}$ el instante de tiempo $t$ en que la CMTC-NH alcanza el estado estacionario. Debido a la dificultad de identificar con precisión dicho valor, se denomina $\widehat{t}^{*}$ un valor $\mathrm{t}$, que por algún tipo de inspección, se considera una aproximación adecuada de $t^{*}$.

\section{E. Representación gráfica de una CMTC}

Un Diagrama de Transición (DT) representa gráficamente las relaciones entre los elementos de una CMTC. Los estados son dibujados como vértices, y las transiciones, por medio de flechas dirigidas desde el vértice del estado i hacia el asociado a $\mathrm{j}$ para una transición de la forma $\mathrm{i} \rightarrow \mathrm{j}$. A cada flecha le corresponde una etiqueta con la tasa $\mathrm{a}_{\mathrm{ij}}(\mathrm{t})$ asociada a dicha transición [12].

\section{F. Solución de CMTC}

Las técnicas desarrolladas para la solución de una CTMC se concentran en resolver el sistema de ecuaciones diferenciales definidas por (4) y (5). Los algoritmos y procedimientos más comunes en la literatura y en la práctica son los métodos numéricos como los algoritmos de Euler y Runge-Kutta, la Transformada de Laplace, la Exponenciación donde se define una función de transición matricial de la forma $\mathbf{P}(\mathrm{t})=$ $\mathrm{e}^{\mathrm{Qt}} \mathbf{P}(0)$, y la Uniformización donde se transforma la CMTC en una Cadena de Markov de Tiempo Discreto análoga [14].

En lo que se refiere a las CMTC-NH, los métodos numéricos, son los más empleados. Estos han demostrado producir los mejores resultados en términos de la precisión y de los recursos computacionales requeridos, especialmente en aquellos casos en que el sistema de ecuaciones diferenciales no posee solución analítica tratable [13]. Normalmente para este fin se emplean softwares matemáticos de propósito general, tales como Matlab y Maple. En ellos, los problemas se traducen como líneas de código, con base en las cuales se ejecutan rutinas o algoritmos para obtener la solución exacta o numérica de precisión dada a través de diferentes tipos de cálculos matemáticos.

\section{Modelos DE DINÁMICA DE SISTEMAS (MDS)}

Matemáticamente, la simbología empleada en el lenguaje de la DS, se traduce en un conjunto de ecuaciones diferenciales que son resueltas recursivamente como funciones del tiempo. Regularmente, estos modelos consisten en un conjunto de variables de nivel que definen el estado del sistema y otro de 
flujo para cada una de ellas [2]. La solución numérica del sistema de ecuaciones equivale a una simulación del modelo, la cual, ejecutada bajo diversos valores de sus parámetros, permiten además analizar la sensibilidad del sistema frente a posibles cambios en las relaciones de los elementos [15].

\section{A. Representación matemática de un MDS}

Considérese un sistema modelado mediante DS que contiene $\mathrm{m}$ variables de nivel, su representación matemática está dada por un conjunto de ecuaciones que corresponden a dos tipos básico: ecuaciones de nivel y ecuaciones de flujo. Aracil [16] describe brevemente cada tipo de ecuaciones, cuyas definiciones se reescriben a conveniencia a continuación.

\section{B. Ecuaciones de nivel}

El valor de una i-ésima variable de nivel $Y(t+\Delta t)_{i}$ en un instante de tiempo dado $t+\Delta t$, es el resultado del flujo neto hacia él, desde un instante inicial t hasta $t+\Delta t$. Cuando $\Delta t$ se hace tan pequeño, que puede ser representado como un elemento diferencial, y $r_{e}(t)_{i}$ y $r_{s}(t)_{i}$ representan respectivamente los flujos instantáneos de entrada y de salida en $\mathrm{t}, \mathrm{Y}(\mathrm{t}+\Delta \mathrm{t})_{\mathrm{i}}$ puede representarse por (7).

$\frac{d Y(t)_{i}}{d x}=r_{e}(t)_{i}-r_{s}(t)_{i}$

\section{Ecuaciones de flujo}

El flujo instantáneo hacia $\mathrm{Y}(\mathrm{t})_{\mathrm{i}}$ es normalmente definido con base en variables exógenas y criterios de relación entre los elementos del sistema. Esto se expresa como:

$r(t)=f\left(Y(t)_{j}, \ldots, c_{j i}(t), \ldots\right) j, i=1, \ldots, m$

Donde $r(t)$ es el valor del flujo neto en el intervalo $[t, t+$ $\Delta t$ ] cuando $\Delta t \rightarrow 0, c_{j i}(t)$ es una función relacionada con el nivel $\mathrm{Y}(\mathrm{t})_{\mathrm{j}}$ y controla el flujo hacia $\mathrm{Y}(\mathrm{t})_{\mathrm{i}}$, yf $(\cdot)$ una función continua dada por las relaciones del sistema. La forma más común de (8) consiste en definir $r(t)$ como combinación de todos los $\mathrm{Y}(\mathrm{t})_{\mathrm{j}}$ mediante las $\mathrm{c}_{\mathrm{ji}}(\mathrm{t})$ correspondientes. De manera que $r_{e}(t)_{i}$ y $r_{s}(t)_{i}$ son de la forma dada por (9) y (10) respectivamente.

$\mathrm{r}_{\mathrm{s}}(\mathrm{t})_{\mathrm{i}}=\sum_{\mathrm{j}=1}^{\mathrm{m}} \mathrm{c}_{\mathrm{ji}}(\mathrm{t}) \mathrm{Y}(\mathrm{t})_{\mathrm{j}}$

$r_{e}(t)_{i}=\sum_{j=1}^{m} c_{j i}(t) Y(t)_{j}$

\section{Representación gráfica de un MDS}

Un MDS es representado gráficamente por medio de un Diagrama de Forrester (DF). Éste consiste en la representación simbólica de los elementos del sistema, haciendo su equivalente conceptual a uno de los tipos de variables definidos y descritos en las Tablas I y II [17].
VARIABles Fundamentales EN Un DF. AdAPTADO DE (BuRns ET AL., 1979)

\begin{tabular}{|c|c|c|c|}
\hline \multicolumn{2}{|c|}{ "VARIABLE } & " SÍMBOLO & DESCRIPCIÓN \\
\hline$Y(t)_{i}$ & Nivel & $Y(t)_{i}$ & $\begin{array}{l}\text { Elemento cuyo valor en el } \\
\text { tiempo es de interés. }\end{array}$ \\
\hline$r(t)_{j i}$ & Flujo & $r(t)_{j i}$ & $\begin{array}{l}\text { Elemento que controla la } \\
\text { transferencia desde el } \\
\text { nivel } j \text { al nivel } i \text {. Cantidad } \\
\text { dinámica. }\end{array}$ \\
\hline$c_{j i}(t)$ & Auxiliar & & $\begin{array}{l}\text { Elemento exógeno } \mathrm{o} \\
\text { intermedio relacionado a } \\
r(t)_{j i} \text { Puede ser } \\
\text { constante o dinámica. }\end{array}$ \\
\hline
\end{tabular}

TABLA II.

Tipo de CONEXIÓN ENTRE LAS VARIABLES DE UN DF. ADAPTADO DE

\begin{tabular}{ccc}
\hline \hline Conexión & Símbolo & Relación \\
\cline { 2 - 3 } Simple & Nivel $\rightarrow$ Flujo \\
& & Nivel $\rightarrow$ Auxiliar \\
& & Auxiliar $\rightarrow$ Flujo \\
Doble & Flujo $\rightarrow$ Nivel \\
& & \\
\hline \hline
\end{tabular}

Para Aracil [16] el DF es una interfaz para la definición de las relaciones matemáticas entre las variables. Estas relaciones están dadas por las ecuaciones de nivel y de flujo, y de otras funciones algebraicas simples, en donde intervienen variables auxiliares, a fin de facilitar la construcción y el análisis del sistema.

\section{EQUIVALENCIA}

En general, la solución de una CTMC-NH consiste en estimar $\overrightarrow{\mathrm{P}}(\mathrm{t})$, ya sea en estado transitorio o estacionario. Esto corresponde a la solución analítica o numérica del sistema compuesto por (4) y (5). Sin embargo, de acuerdo con (2), las funciones de intensidad (componentes de $\mathbf{A}(\mathrm{t})$ ) dependen de $\mathrm{t}$, de manera que hallar tal solución no es una tarea fácil. Especialmente, en la medida que la naturaleza de $\mathrm{a}_{\mathrm{ji}}(\mathrm{t})$ toma expresiones más complejas y se incrementa el número de estados del proceso estocástico [13]. Desde el punto de vista analítico, puede incluso no existir solución, de manera que se requieren aproximaciones numéricas a través de recursos computacionales [14].

Una solución de este último tipo se puede obtener al construir un Modelo de Dinámica de Sistemas Equivalente (MDSE) para una CMTC-NH. Dicho modelo se basa en el desarrollo de la siguiente equivalencia.

Sea el sistema de ecuaciones diferenciales definido por (4), ésta se puede escribir como:

$$
\frac{d P_{i}(t)}{d t}=a_{i i}(t) P_{i}(t)+\sum_{\substack{j \neq i \\ j=1}}^{k} a_{j i}(t) P_{j}(t) \forall i
$$

Luego, reemplazando $\mathrm{a}_{\mathrm{ii}}(\mathrm{t})$ de (3), (11) es equivalente a:

TABLA I

$$
\frac{d P_{i}(t)}{d t}=-\sum_{\substack{j \neq i \\ j=1}}^{k} a_{i j} P_{i}(t)+\sum_{\substack{j \neq i \\ j=1}}^{k} a_{j i}(t) P_{j}(t) \forall i
$$


Ahora, recordando las representaciones matemáticas de un MDS dadas en las Ecuaciones 7 y 8 , y haciendo $\mathrm{k}=\mathrm{m}, \mathrm{P}_{\mathrm{i}}(\mathrm{t})=$ $\mathrm{Y}(\mathrm{t})_{\mathrm{i}}, \quad \mathrm{a}_{\mathrm{ij}}(\mathrm{t})=\mathrm{c}_{\mathrm{ij}}(\mathrm{t}), \quad \mathrm{ya}_{\mathrm{ji}}(\mathrm{t})=\mathrm{c}_{\mathrm{ji}}(\mathrm{t})$, se tiene que (12) es equivalente a:

$$
\frac{d Y(t)_{i}}{d t}=-\sum_{\substack{j \neq 1 \\ j=1}}^{m} c_{i j} Y(t)_{i}+\sum_{\substack{j \neq i \\ j=1}}^{m} c_{j i}(t) Y(t)_{j}
$$

Si se permite el flujo de entrada al nivel $\mathrm{Y}(\mathrm{t})_{\mathrm{i}}$ ser función de los demás niveles $Y(t)$, con $\mathrm{j} \neq \mathrm{i}$; y el flujo de salida de $\mathrm{Y}(\mathrm{t})_{\mathrm{i}}$ ser función única y exclusivamente de sí mismo, (9) y (10) se reducen a:

$$
\begin{aligned}
& r_{s}(t)_{i}=\sum_{\substack{j \neq 1 \\
j=1}}^{m} c_{i j}(t) Y(t)_{i} \\
& r_{e}(t)_{i}=\sum_{\substack{j \neq i \\
j=1}}^{m} c_{j i}(t) Y(t)_{j}
\end{aligned}
$$

La Ecuación 13 es una ecuación de nivel, tal como está definida en (7). De aquí se concluyen las siguientes correspondencias:

a) $\mathrm{P}_{\mathrm{i}}(\mathrm{t})$ puede ser modelada por medio de una variable de nivel $\mathrm{Y}(\mathrm{t})_{\mathrm{i}}$ en un MDS.

b) $\sum_{\substack{j \neq i \\ j=1}}^{k} a_{i j}(t) P_{i}(t)$ es equivalente a $r_{s}(t)_{i}$, mientras $\sum_{\substack{j \neq i \\ j=1}}^{k} a_{j i}(t) P_{j}(t)$ lo es a $r_{e}(t)_{i}$ y pueden ser modeladas por medio de flujos en un MDS.

La construcción de un MDSE consiste en hacer las anteriores correspondencias, las cuales se pueden resumir conceptualmente al decir: "la probabilidad de que $\{X(t), t \geq$ $0\}$ esté en un estado $\mathrm{i} \in \mathrm{E}$, es igual al valor de una variable de nivel cuyos flujos de entrada y salida son, de acuerdo con (14) y (15), combinaciones de los otros estados $j(j \neq i)$ para el primer caso, y de sólo sí mimo en el segundo."

Un factor importante a tener en cuenta es definir la variable $t$, el periodo corrido de simulación, como una variable explícita, condición que diferencia un MDSE de los MDS comunes, en los que el tiempo es implícito. Esto se logra al crear una variable de nivel denominada $t$ que integra el valor constante de 1 y con valor inicial igual a 0 .

El proceso de construcción de un MDSE se presenta en el diagrama ilustrado en la Fig. 1.

\section{A. Algunos aspectos sobre un MDSE.}

Normalmente en los MDS, es de interés el análisis de los patrones de comportamiento, más que los resultados numéricos de la simulación. Lo contrario ocurre regularmente en aplicaciones de sistemas físicos en los cuales interesa en valor de las variables en instantes de tiempo dado [8]. El desarrollo de un MDSE tiene esta necesidad. El empleo de procesos estocásticos tiene como fin conocer la posible evolución de un conjunto de eventos, de los cuales se desea conocer su probabilidad de ocurrencia ya sea en caso transitorio o estacionario. De aquí, que se deba garantizar la mayor precisión posible en las aproximaciones numéricas obtenidas.

Los softwares de DS, tales como VensimeIThink/Stella, emplean métodos numéricos para la simulación del sistema. Vensim, por ejemplo, dispone de tres métodos de integración.

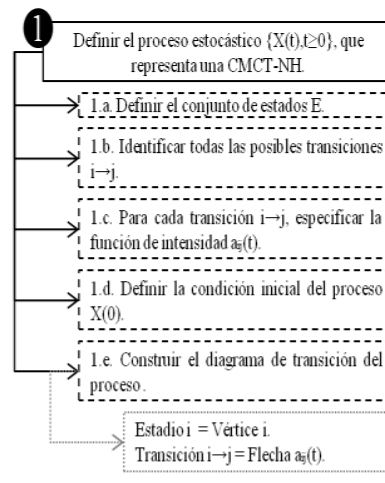

2 Realizar la conversión gráicica desde el diagram de transición a un diagrama de Forrester: $\rightarrow$ 2.a. Redibujar cada vétice i como una I variable de nivel llamada $P_{i}(t)$ 2.b. Redibujar cada fecha dirigida aj(t) como I una variable flujo llamada rist), que une $P(t)$ $\rightarrow$ una variableflo ihe Iy $P_{j}(t)$ mediante tuna conexion de flecha idoble. i 2 c. Dibujar flechas simples desde cada nivel L P.(t) hacia todos sus flujos de salida $\mathrm{I}_{\mathrm{j}}(\mathrm{t})$. ${ }^{2}$ d. Dibujar cada af(t) como una variable 1 auxiliar

L.-.-. 2.e. Dibujar flechas simples desde cada $>_{1}$ variable auxiliar $a_{j}(t)$ hacia el flujo $r_{j}(t)$ correspondiente. 2f Dibujar una variable de nivel $\rightarrow$ denominada t, y unirla con flechas simples haciatodas las variables as $(t)$.

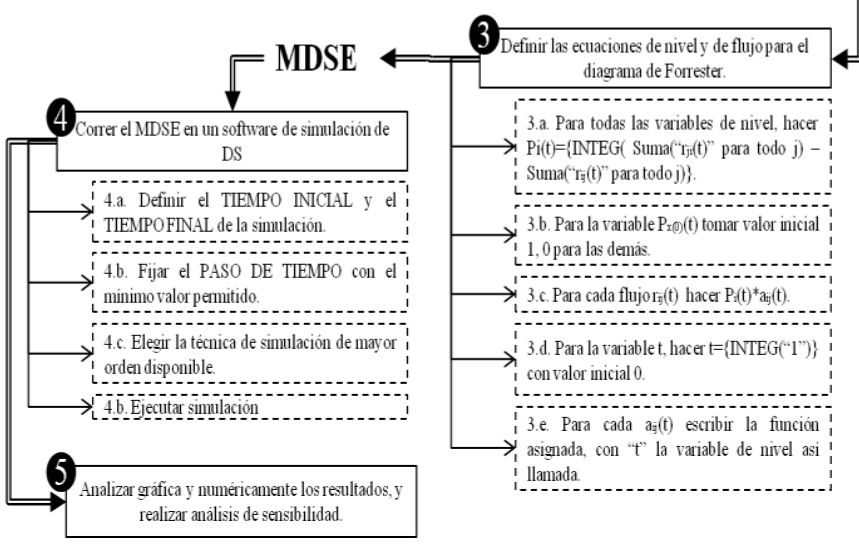

Fig. 1. Proceso de construcción de un MDSE.

Método de Euler, Ecuaciones en Diferencia y Métodos Runge-Kutta (RK). Entre estos últimos la técnica más popular y eficiente al minimizar el error de truncamiento global en las estimaciones es el método RK de Cuarto Orden (RK4). Este error depende básicamente de la longitud del paso de integración empleado, el cual es equivalente al PASO DE TIEMPO definido en el programa. De aquí, que la simulación de una CMTC-NH en software de DS requiera el empleo del método de integración disponible con el menor error de truncamiento, así como el uso del menor PASO DE TIEMPO (tamaño del intervalo entre estimaciones numéricas sucesivas del algoritmo de aproximación) posible, a fin de garantizar el mayor nivel de precisión en la estimación numérica, y el supuesto de continuidad de $t$.

Adicionalmente, el desarrollo de un MDSE mediante dichos software, permite realizar otros tipos de análisis que no son posibles en software matemáticos como Maple y Matlab al solucionar el sistema generado por (4) y (5). La interfaz y las 
herramientas disponibles en VenSim y en otros softwares de DS, permiten un rápido análisis gráfico de los resultados, su exportación, y principalmente, ejecutar diferentes simulaciones a fin de analizar la sensibilidad del sistema.

Teniendo en cuenta las ventajas anteriores, el MDSE facilita estimar e identificar características de la CMTC que representa, tales como: la distribución de probabilidad tanto en el periodo transitorio como estacionario, y el instante de tiempo $\widehat{t}^{*}$ en que se estima inicia dicho periodo de estabilidad.

\section{DesarRollo de Un MDSE.}

Se desea analizar un proceso estocástico que satisface la propiedad markoviana, con transiciones entre dos estados accesibles uno del otro. A continuación se representa la descripción de cada uno de los pasos definidos en el diagrama de flujo de la Fig. 1.

1. Considere una CMTC - NH, $\{\mathrm{X}(\mathrm{t}), \mathrm{t} \geq 0\}$ con las siguientes características:

- El conjunto de estados está dado por $\{1,2\}$.

- Puesto que ambos estados se comunican entre sí, son posibles las transiciones $1 \rightarrow 2$ y $2 \rightarrow 1$.

- Las funciones de intensidad matricial son funciones lineales del tiempo, dadas por $\mathrm{a}_{12}(\mathrm{t})=\mathrm{t} / 10 \mathrm{y}$ $\mathrm{a}_{21}(\mathrm{t})=\mathrm{t} / 20$. De acuerdo con (3), se tiene que $a_{11}(t)=-t / 10 \quad$ y $\quad a_{22}(t)=-t / 20$. En forma matricial éstas se presentan como $\mathbf{A}(\mathrm{t})=$ $\left[\begin{array}{cc}-t / 10 & t / 10 \\ t / 20 & -t / 20\end{array}\right]$

- La distribución de probabilidad inicial es $\overrightarrow{\mathrm{P}}(0)=$ $(1,0)$.

- El diagrama de transición se muestra en la Fig. 2.

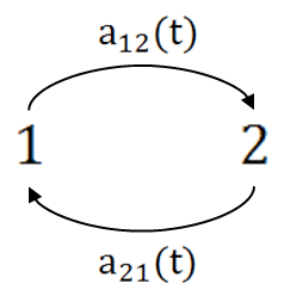

Fig. 2. Diagrama de transición de una CMTC-NH con dos estados

2. El DF equivalente a $\{X(t), t \geq 0\}$ se ilustra en la Fig. 3.

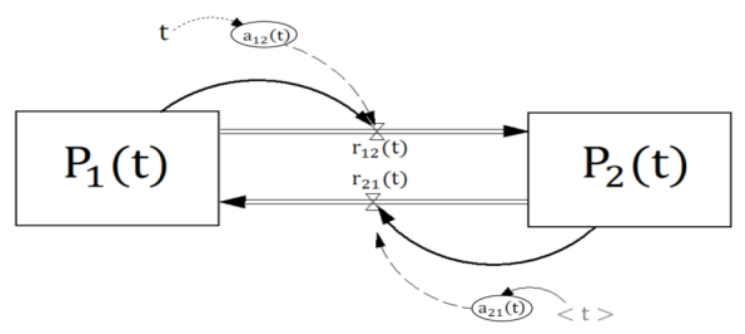

Fig. 3. DF para una CMTC-NH con dos estados

3. A continuación se presentan el conjunto de ecuaciones que se asignan a cada variable.
$\mathrm{P}_{1}(\mathrm{t}):\left\{=\operatorname{INTEG}\left(" \mathrm{r}(\mathrm{t})_{21} "-\mathrm{r}(\mathrm{t})_{12}\right.\right.$ "); Valor Inicial $=$ $1 ;$ Tipo $=$ Nivel $\}$;

$\mathrm{P}_{2}(\mathrm{t}):\left\{=\operatorname{INTEG}\left(" \mathrm{r}(\mathrm{t})_{12} "-\mathrm{r}(\mathrm{t})_{21} "\right)\right.$; Valor Inicial $=$ 0 ; Tipo $=$ Nivel $\}$;

$\mathrm{r}(\mathrm{t})_{12}:\left\{" \mathrm{P}_{1}(\mathrm{t}) " *\right.$ " $\mathrm{a}_{12}(\mathrm{t}) " ;$ Tipo = Flujo $\}$

$\mathrm{r}(\mathrm{t})_{21}:\left\{" \mathrm{P}_{2}(\mathrm{t}) " *\right.$ * $\mathrm{a}_{21}(\mathrm{t}) " ;$ Tipo = Flujo $\}$;

$\mathrm{a}_{12}(\mathrm{t}):\{" \mathrm{t} " / 10 ;$ Tipo $=$ Auxiliar $\} ;$

$\mathrm{a}_{21}(\mathrm{t}):\{\mathrm{t}$ " $/ 20 ;$ Tipo $=$ Auxiliar $\}$;

t: $\{=$ INTEG("1"); Valor Inicial = 0; Tipo = Nivel $\}$.

4. La simulación del MDSE construido se realiza en el software de DS VenSim Professional Versión 5.3, con los siguientes parámetros: TIEMPO INICIAL $=0$; TIEMPO FINAL $=20 ;$ PASO DE TIEMPO $=0.0078125$; Técnica de simulación: RK4 Auto.

5. La Fig. 4 ilustra el comportamiento de las probabilidades de estado para el ejemplo desarrollado. Como es de esperarse, dichas probabilidades varían a través del tiempo hasta que alcanzan $\widehat{t}^{*}$, instante a partir del cual permanecen constantes hasta que alcanza $\mathrm{t}=$ TIEMPO FINAL.

Haciendo caso a (6), y considerando que $t=20$ representa un instante de tiempo lo suficientemente grande, se puede decir que los resultados siguientes corresponden a las probabilidades en estado estacionario: $\mathrm{P}_{1}=\mathrm{P}_{1}(20)=$ 0.333333 y $\mathrm{P}_{2}=\mathrm{P}_{2}(20)=0.666667$.
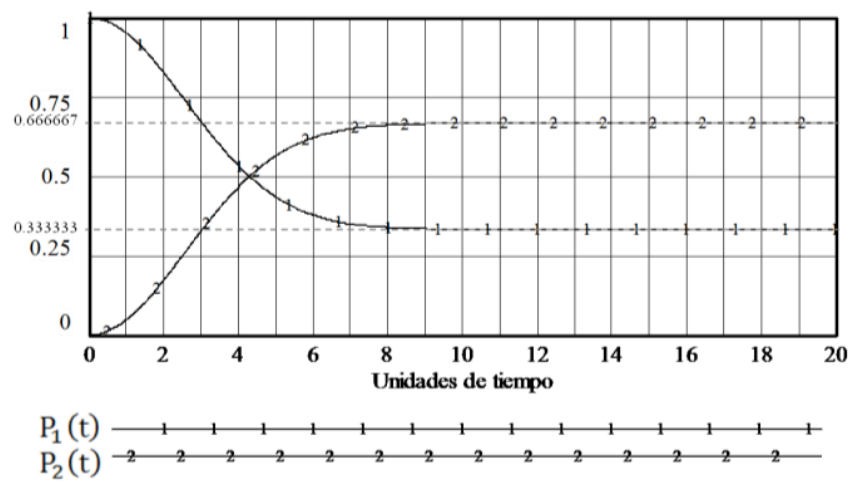

Fig. 4. Funciones de las probabilidades de estado para una CTMC-NH de dos estados.

Cuando el software de DS empleado permite la exportación de los datos de la simulación, se puede obtener un valor de $\widehat{t^{*}}$ al calcular las pendientes de las funciones de probabilidad de estado para cada par de periodos consecutivos. La Fig. 5 presenta la pendiente de las funciones de probabilidad para los estados del ejemplo desarrollado. Como se observa, después del $t=10$, la pendiente es aproximadamente 0 , de manera que para cualquier periodo de tiempo $t>10$, no hay variación en la probabilidad de estado y se puede considerar que el proceso ha alcanzado su estado estacionario, de manera que $\widehat{\mathrm{t}^{*}}=10$. Cuando no es posible realizar este tipo de cálculos con los 
datos de la simulación, $\widehat{t^{*}}$ puede ser aproximado por inspección visual de las gráficas generadas para las variables de nivel que representan las probabilidades de estado.

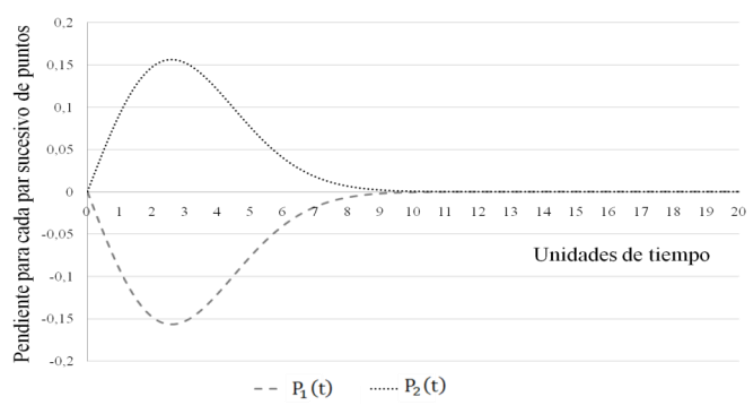

Fig. 5. Pendiente de las funciones de probabilidad de estado para una CMTC$\mathrm{NH}$ de dos estados

Además, a modo de ilustración, la Fig. 6 presenta las funciones de probabilidad de $\mathrm{P}_{1}(\mathrm{t})$ cuando $\mathrm{a}_{12}(\mathrm{t})=\mathrm{t} / 10 \mathrm{y}$ $\mathrm{a}_{12}{ }^{*}(\mathrm{t})=\mathrm{t} / 2$. Este tipo de comparación es posible en VenSim mediante la ejecución de simulaciones independientes, cambiando en cada una, la función de intensidad. Las herramientas gráficas disponibles, permiten además una rápida inspección de los cambios en el sistema, actividad que, realizada en otro tipo de software, requeriría para cada variación, obtener la solución como si se tratara de un nuevo problema y llevar a cabo de nuevo todos los pasos requeridos para ello.
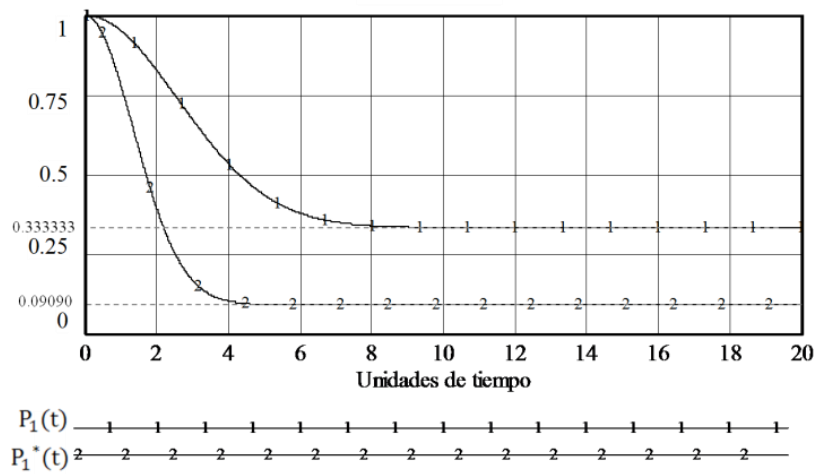

Fig. 6. Análisis de sensibilidad respecto a $\mathrm{a}_{12}(\mathrm{t})$ para una CMTC-NH de dos estados

En el ejemplo, es claro que el aumento de la función de intensidad $\mathrm{a}_{12}(\mathrm{t})$ conduce a que el proceso tenga una mayor probabilidad de estar en el estado 2 a través del tiempo. De aquí que la probabilidad de estado estacionario sea $\mathrm{P}_{1}{ }^{*}=$ 0.0909 , representado una disminución del $72,3 \%$ respecto al valor original.

\section{A. Validación numérica}

Como se ha dicho, el principal objetivo al resolver una CMTC es la estimación de la función de distribución $\overrightarrow{\mathrm{P}}(\mathrm{t})$, para ello se emplean las técnicas descritas en la sección II-F. La construcción de un MDSE en un software de DS permite realizar dichas estimaciones a partir de la técnica de aproximación numérica empleada para la simulación, de ahí la importancia de la elección del método numérico y del PASO DE TIEMPO. Para demostrar que bajo las especificaciones dadas para las dos decisiones anteriores, la estimación obtenida por medio de la simulación del MDSE es aceptable, se compara con la solución exacta obtenida mediante el software Maple 14.00.

Esta solución analítica resulta de solucionar el sistema de ecuaciones diferenciales definido en las Ecuaciones 4 y 5 . Para el ejemplo desarrollado dicho sistema queda dado por (16), (17) y (18) dadas a continuación con $\mathrm{P}_{1}(0)=1 \mathrm{y}$ $\mathrm{P}_{2}(0)=0$.

$$
P_{1}(t)+P_{2}(t)=1
$$

$\frac{d P_{1}(t)}{d t}=-(t / 10) P_{1}(t)+(t / 20) P_{2}(t)$

$\frac{d P_{2}(t)}{d t}=(t / 10) P_{1}(t)+(-t / 20) P_{2}(t)$

La solución del sistema anterior se especifica en (19) y (20).

$P_{1}(t)=\frac{1}{3}+\frac{2}{3} \exp \left(-\frac{3 t^{2}}{40}\right)$

$\mathrm{P}_{2}(\mathrm{t})=\frac{2}{3}-\frac{2}{3} \exp \left(-\frac{3 \mathrm{t}^{2}}{40}\right)$

Sin embargo, debe tenerse en cuenta, que hallar este tipo de expresiones para la solución de un sistema de ecuaciones diferenciales, no es siempre posible. En el ejemplo, gracias al número de variables y a la naturaleza lineal de las funciones de intensidad, se encuentran expresiones relativamente simples, en otros casos la solución analítica no existe y exigen el uso de los métodos numéricos como el empleado por el software de DS.

En la Tabla III se muestran los valores correspondientes a las probabilidades $\mathrm{P}_{1}(\mathrm{t})$ y $\mathrm{P}_{2}(\mathrm{t})$ para 20 valores de $\mathrm{t}$, estos valores se calcularon con el modelo de simulación propuesto y también a partir de la solución con software matemático para las (19) y (20). Además, en la Tabla III muestra los valores correspondientes al error absoluto y relativo frente al valor teórico. Se observa entonces que las diferencias entre uno y otro se encuentran más allá de la quinta cifra significativa, valor que se puede considerar adecuado en las aplicaciones prácticas. 
COMPARACIÓN ENTRE LA SOLUCIÓN ANALÍTICA Y LA NUMPERICA OBTENIDA DESDE Un MDSE PARA UN MM

\begin{tabular}{|c|c|c|c|c|c|c|c|c|}
\hline \multirow{2}{*}{$\mathrm{t}$} & \multicolumn{2}{|c|}{ Analítica } & \multicolumn{2}{|c|}{ MDSE } & \multicolumn{2}{|c|}{$\begin{array}{c}\text { Error } \\
\text { Absoluto }\end{array}$} & \multicolumn{2}{|c|}{$\begin{array}{c}\text { Error } \\
\text { Relativo }\end{array}$} \\
\hline & P1 & P2 & P1 & P2 & P1 & P2 & P1 & P2 \\
\hline 0 & $\begin{array}{c}1,00000 \\
0\end{array}$ & & $\begin{array}{c}1,00000 \\
0\end{array}$ & & & & & \\
\hline 1 & $\begin{array}{c}0,95182 \\
9\end{array}$ & $\begin{array}{c}0,04817 \\
1\end{array}$ & $\begin{array}{c}0,95182 \\
9\end{array}$ & $\begin{array}{c}0,04817 \\
1\end{array}$ & $\begin{array}{c}0,00000 \\
0\end{array}$ & $\begin{array}{c}0,00000 \\
0\end{array}$ & 0,0000 & 0,0000 \\
\hline 2 & $\begin{array}{c}0,82721 \\
2\end{array}$ & $\begin{array}{c}0,17278 \\
8\end{array}$ & $\begin{array}{c}0,82721 \\
1\end{array}$ & $\begin{array}{c}0,17278 \\
8\end{array}$ & $\begin{array}{c}- \\
0,00000 \\
1\end{array}$ & $\begin{array}{c}0,00000 \\
0\end{array}$ & $-0,0001$ & $-0,0001$ \\
\hline 3 & $\begin{array}{c}0,67277 \\
1\end{array}$ & $\begin{array}{c}0,32722 \\
9\end{array}$ & $\begin{array}{c}0,67277 \\
0\end{array}$ & $\begin{array}{c}0,32722 \\
9\end{array}$ & $\underset{1}{-}$ & $\begin{array}{c}0,00000 \\
0\end{array}$ & $-0,0001$ & $-0,0001$ \\
\hline 4 & $\begin{array}{c}0,53412 \\
9\end{array}$ & $\begin{array}{c}0,46587 \\
1\end{array}$ & $\begin{array}{c}0,53412 \\
9\end{array}$ & $\begin{array}{c}0,46587 \\
0\end{array}$ & $\underset{1}{-}$ & $\begin{array}{c}- \\
0,00000 \\
1\end{array}$ & $-0,0001$ & $-0,0001$ \\
\hline 5 & $\begin{array}{c}0,43557 \\
0\end{array}$ & $\begin{array}{c}0,56443 \\
0\end{array}$ & $\begin{array}{c}0,43556 \\
9\end{array}$ & $\begin{array}{c}0,56442 \\
9\end{array}$ & $\begin{array}{c}0,00000 \\
1\end{array}$ & $\underset{1}{0,00000}$ & $-0,0002$ & $-0,0002$ \\
\hline 6 & $\begin{array}{c}0,37813 \\
7\end{array}$ & $\begin{array}{c}0,62186 \\
3\end{array}$ & $\begin{array}{c}0,37813 \\
7\end{array}$ & $\begin{array}{c}0,62186 \\
2\end{array}$ & $\begin{array}{c}0,00000 \\
0\end{array}$ & $\begin{array}{c}- \\
0,00000 \\
1\end{array}$ & $-0,0001$ & $-0,0001$ \\
\hline 7 & $\begin{array}{c}0,35023 \\
3\end{array}$ & $\begin{array}{c}0,64976 \\
7\end{array}$ & $\begin{array}{c}0,35023 \\
2\end{array}$ & $\begin{array}{c}0,64976 \\
6\end{array}$ & $\begin{array}{c}0,00000 \\
1\end{array}$ & $\underset{1}{0,00000}$ & $-0,0002$ & $-0,0002$ \\
\hline 8 & $\begin{array}{c}0,33882 \\
0\end{array}$ & $\begin{array}{c}0,66118 \\
0\end{array}$ & $\begin{array}{c}0,33881 \\
9\end{array}$ & $\begin{array}{c}0,66117 \\
9\end{array}$ & $\begin{array}{c}- \\
0,00000 \\
1\end{array}$ & $\begin{array}{c}- \\
0,00000 \\
1\end{array}$ & $-0,0002$ & $-0,0002$ \\
\hline 9 & $\begin{array}{c}0,33486 \\
6\end{array}$ & $\begin{array}{c}0,66513 \\
4\end{array}$ & $\begin{array}{c}0,33486 \\
6\end{array}$ & $\begin{array}{c}0,66513 \\
3\end{array}$ & $\begin{array}{c}- \\
0,00000 \\
1\end{array}$ & $\begin{array}{c}- \\
0,00000 \\
1\end{array}$ & $-0,0001$ & $-0,0001$ \\
\hline 10 & $\begin{array}{c}0,33370 \\
2\end{array}$ & $\begin{array}{c}0,66629 \\
8\end{array}$ & $\begin{array}{c}0,33370 \\
1\end{array}$ & $\begin{array}{c}0,66629 \\
7\end{array}$ & $\begin{array}{c}- \\
0,00000 \\
1\end{array}$ & $\underset{0,00000}{-}$ & $-0,0002$ & $-0,0002$ \\
\hline 11 & $\begin{array}{c}0,33341 \\
0\end{array}$ & $\begin{array}{c}0,66659 \\
0\end{array}$ & $\begin{array}{c}0,33340 \\
9\end{array}$ & $\begin{array}{c}0,66658 \\
9\end{array}$ & $\begin{array}{c}- \\
0,00000 \\
1\end{array}$ & $\begin{array}{c}- \\
0,00000 \\
1\end{array}$ & $-0,0002$ & $-0,0002$ \\
\hline 12 & $\begin{array}{c}0,33334 \\
7\end{array}$ & $\begin{array}{c}0,66665 \\
3\end{array}$ & $\begin{array}{c}0,33334 \\
6\end{array}$ & $\begin{array}{c}0,66665 \\
2\end{array}$ & $\begin{array}{c}- \\
0,00000 \\
1\end{array}$ & $\begin{array}{c}- \\
0,00000 \\
1\end{array}$ & $-0,0001$ & $-0,0001$ \\
\hline 13 & $\begin{array}{c}0,33333 \\
5\end{array}$ & $\begin{array}{c}0,66666 \\
5\end{array}$ & $\begin{array}{c}0,33333 \\
4\end{array}$ & $\begin{array}{c}0,66666 \\
2\end{array}$ & $\underset{1}{0,00000}$ & $\underset{0,00000}{-}$ & $-0,0003$ & $-0,0003$ \\
\hline 14 & $\begin{array}{c}0,33333 \\
4\end{array}$ & $\begin{array}{c}0,66666 \\
6\end{array}$ & $\begin{array}{c}0,33333 \\
4\end{array}$ & $\begin{array}{c}0,66666 \\
2\end{array}$ & $\begin{array}{c}0,00000 \\
0\end{array}$ & $\begin{array}{c}- \\
0,00000 \\
4\end{array}$ & $-0,0006$ & $-0,0006$ \\
\hline 15 & $\begin{array}{c}0,33333 \\
3\end{array}$ & $\begin{array}{c}0,66666 \\
7\end{array}$ & $\begin{array}{c}0,33333 \\
4\end{array}$ & $\begin{array}{c}0,66666 \\
2\end{array}$ & $\begin{array}{c}0,00000 \\
0\end{array}$ & $\underset{4}{0,00000}$ & $-0,0007$ & $-0,0007$ \\
\hline 16 & $\begin{array}{c}0,33333 \\
3\end{array}$ & $\begin{array}{c}0,66666 \\
7\end{array}$ & $\begin{array}{c}0,33333 \\
3\end{array}$ & $\begin{array}{c}0,66666 \\
2\end{array}$ & $\begin{array}{c}0,00000 \\
0\end{array}$ & $\underset{4}{0,00000}$ & $-0,0007$ & $-0,0007$ \\
\hline 17 & $\begin{array}{c}0,33333 \\
3\end{array}$ & $\begin{array}{c}0,66666 \\
7\end{array}$ & $\begin{array}{c}0,33333 \\
3\end{array}$ & $\begin{array}{c}0,66666 \\
2\end{array}$ & $\begin{array}{c}0,00000 \\
0\end{array}$ & $\begin{array}{c}- \\
0,00000 \\
4\end{array}$ & $-0,0007$ & $-0,0007$ \\
\hline 18 & $\begin{array}{c}0,33333 \\
3\end{array}$ & $\begin{array}{c}0,66666 \\
7\end{array}$ & $\begin{array}{c}0,33333 \\
3\end{array}$ & $\begin{array}{c}0,66666 \\
2\end{array}$ & $\begin{array}{c}0,00000 \\
0\end{array}$ & $\begin{array}{c}- \\
0,00000 \\
4\end{array}$ & $-0,0007$ & $-0,0007$ \\
\hline 19 & $\begin{array}{c}0,33333 \\
3\end{array}$ & $\begin{array}{c}0,66666 \\
7\end{array}$ & $\begin{array}{c}0,33333 \\
3\end{array}$ & $\begin{array}{c}0,66666 \\
2\end{array}$ & $\begin{array}{c}0,00000 \\
0\end{array}$ & $\underset{4}{-}$ & $-0,0007$ & $-0,0007$ \\
\hline 20 & $\begin{array}{c}0,33333 \\
3\end{array}$ & $\begin{array}{c}0,66666 \\
7\end{array}$ & $\begin{array}{c}0,33333 \\
3\end{array}$ & $\begin{array}{c}0,66666 \\
2\end{array}$ & $\begin{array}{c}0,00000 \\
0\end{array}$ & $\underset{4}{0,00000}$ & $-0,0007$ & $-0,0007$ \\
\hline
\end{tabular}

\section{CONCLUSIONES}

Los MDSE trabajan con dos tipos diferentes de modelación: las CMTC correspondientes a procesos estocásticos, y los MDS que tratan con sistemas con relaciones determinísticas bien definidas. El temor es que modelar una CMTC mediante un MDS, podría dar la falsa impresión de que el sistema y su evolución se está modelando como un proceso determinista, sino se tiene bien entendido cual es el significado de las variables de nivel y de las variables de flujo dentro del proceso markoviano.

A pesar de este potencial error, la construcción de un MDSE es un concepto, que bien empleado presenta las siguientes ventajas:

- Los conceptos asociados a los MDS parecen ser mejor y más fácilmente entendidos que los asociados a las CMTC.
De aquí, que en términos pedagógicos la equivalencia planteada supone la oportunidad de integrar ambas metodologías con el fin de enriquecer una con otra. Por un lado, porque los MDS, confinados generalmente a relaciones determinísticas, pueden ser ampliados con relaciones probabilísticas para tratar con la incertidumbre en los modelos; y por otro, porque las herramientas de la DS pueden facilitar el proceso de construcción y de análisis de un MM gracias a las herramientas gráficas y de sensibilidad disponibles en el software de DS.

- La solución analítica de las CMTC-NH pueden ser, sino en todas, en la mayoría de los casos intratable, por lo cual el análisis tiende a concentrarse en las probabilidades de estado estacionario. Por su parte, la modelación con DS, se concentra en general, en el comportamiento transitorio del sistema sobre toda la escala de tiempo elegida, esto se logra porque el software de DS permite realizar simulaciones en todo el horizonte de análisis elegido obteniendo estimaciones numéricas en intervalos de tiempo definidos. Entendiendo que conocer cuál estado va a prevalecer en el futuro, es igual de importante que conocer la respuesta transitoria del sistema, la construcción de un MDSE permite analizar la CMTC sin restricciones en el dominio del tiempo en todo el horizonte seleccionado.

- La equivalencia demostrada entre una CMTC-NH y un MDS puede motivar el empleo del primer tipo de modelo. Esto, porque gran parte de su aplicación limitada se debe a la dificultad de computación y de cálculo numérico, restricciones eliminadas gracias al uso del software para DS. Se puede, por tanto, modelar procesos con funciones de intensidad de diferente tipo y realizar análisis que en software matemático no está disponible o es de relativa complejidad.

Una pregunta natural frente a lo descrito anteriormente es si la construcción de un MDSE es siempre adecuado. De acuerdo con los esfuerzos de elaboración, los potenciales errores, y los beneficios obtenidos, se puede decir que el empleo de un MSDE resulta útil cuando se trata con sistemas relativamente complejos, y que a diferencia de las CTMC-H requieren la incorporación de otros elementos cuyo análisis presente alto nivel de complejidad en otro tipo de herramientas. Por el contrario, si el análisis de concentra en procesos con solución analítica disponible, probablemente no haya una razón importante que justifique el empleo de la equivalencia.

El concepto de un MDSE queda a disposición de los modeladores y su empleo depende básicamente del nivel de complejidad del MM y la disponibilidad de recursos para solucionarlo. Se espera, por tanto, que la equivalencia teórica y el procedimiento de construcción descrito e ilustrado, enriquezca los trabajos futuros tanto en el campo de los procesos estocásticos como en la modelación con DS. 


\section{REFERENCIAS}

[1] H. Nuhoglu and M. Nuhoglu, "System dynamics approach in science and technology education," J. Turkish Sci. Educ., vol. 4, no. 2, pp. 91-108, 2007.

[2] Y.-T. Chen and B. Jeng, "Yet another Representation for System Dynamics Models, and Its Advantages," in System Dynamics Society Conference, 2002.

[3] H. Tijms, A First Course n Stochastic Models. 2003. DOI: 10.1002/047001363X

[4] K. Sahin, "System Dynamics Models: Some Obscurities," IEEE Trans. Syst. Man. Cybern., vol. 9, no. 2, pp. 91-93, 1979.

[5] K. Sahin, "Equivalence of Markov Models to a Class of System Dynamics Models," IEEE Trans. Syst. Man. Cybern., vol. 9, no. 7, pp. 398-402, 1979.

[6] D. Wood, "Modeling Stochastic Processes with System Dynamics," in Proceedings of the 1983 System Dynamics Conference, 1983.

[7] E. Mosekilde and S. Rasmussen, "Random Processes in System Dynamics," Dynamica, vol. 9, no. 1, pp. 33-39, 1983.

[8] P. K. J. Mohapatra and R. K. Roy, "System Dynamics Models for Markov Processes," Proceedings of the 1991 International System Dynamics Conference: System Dynamics '91 Supplement: 1991.

[9] M. S. Rao and V. N. A. Naikan, "A Novel Markov System Dynamics Framework for Reliability Analysis of Systems," Econ. Qual. Control, vol. 24, pp. 101-116, 2010. DOI: 10.1515/EQC.2009.101

[10] S. M. Ross, Introduction to Probability Models. 2007.

[11] J. Jacques and M. Raimondo, Semi-markov risk models for finance, insurance and reliability. 2007.

[12] D. Sherwin, A. Hoyland, and M. Rausand, "System Reliability Theory-Models and Statistical Methods.," Stat., p. 636, 1995. DOI: $10.2307 / 2348906$

[13] T. Zhang and M. Horigome, "Availability and reliability of system with dependent components and time-varying failure and repair rates," IEEE Trans. Reliab., vol. 50, no. 2, pp. 151-158, 2001. DOI: $10.1109 / 24.963122$

[14] M. Arns, P. Buchholz, and A. Panchenko, "On the numerical analysis of inhomogeneous continuous-time Markov chains," INFORMS J. Comput., vol. 22, no. 3, pp. 416-432, 2010. DOI: 10.1287/ijoc.1090.0357

[15] T. Rolski, H. Schmidli, V. Schmidt, and J. Teugels, Stochastic Processes for Insurance and Finance. 2008. DOI: 10.1002/9780470317044

[16] J. Aracil, Dinámica de Sistemas, 1st ed. Madrid: Isdefe, 1995.

[17] J. R. Burns, O. M. Ulgen, and H. W. Beights, "An Algorithm for Converting Signed Digraphs to Forrester Schematics," IEEE Trans. Syst. Man Cybern., vol. 9, no. 3, pp. 115-124, 1979.

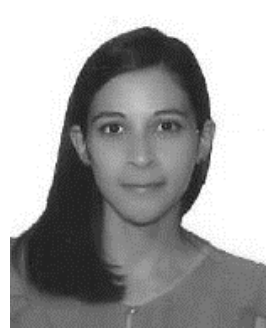

Marcela Narváez Velasco, nacida el 23 de diciembre en Cali, Colombia. Ingeniera Industrial egresada de la Universidad del Valle, Santiago de Cali, Colombia, en 2012. Magister en Finanzas de la Universidad Icesi, Santiago de Cali Colombia, en 2018.
Entre los años 2012-2016, desempeñó cargos de coordinación en procesos de compras y abastecimiento de las empresas Compañía Internacional de Alimentos S.A.S y Fleischmann Foods SA. Actualmente es consultora funcional de finanzas en proyectos de implementación y soporte de aplicaciones Oracle en Iteria SAS.

ORCID: https://orcid.org/0000-0001-7461-2139

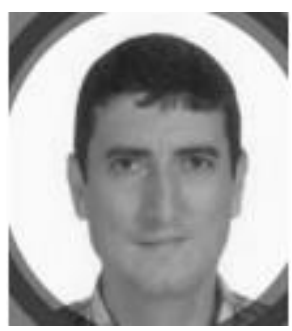

J.C. Osorio-Gómez, nacido el 20 de septiembre de 1975 en Buga, Valle del Cauca, Colombia. Ingeniero Industrial, Especialista en Logística, Magister en Ingeniería Industrial, Doctor en Ingeniería Industrial de la Universidad del Valle, Santiago de Cali, Valle del Cauca, Colombia. Actualmente Profesor titular de la Escuela de Ingeniería Industrial de la Universidad del Valle.

ORCID: http://orcid.org/0000-0001-5625-5609 